\title{
Serial reversal learning in the mallard duck (Anas platyrhynchos)
}

\author{
MICHAEL C. WELLS and PHILIP N. LEHNER \\ Colorado State University, Fort Collins, Colorado 80523
}

\begin{abstract}
Serial reversal learning problems have been used primarily with the pigeon to develop theories of learning for the class Aves as a whole. This experiment tested the mallard duck and employed a procedure similar to that used for $t$ : e pigeon. Five mallard ducks were tested in a visual discrimination task in daily sessions, with the rewarded symbol changed every 20 days. Improvement in the performance of the ducks did not occur to the same degree as that reported for the pigeon, and no evidence was found for a weakening of retention over time.
\end{abstract}

Serial reversal learning problems have been used to demonstrate that various species differ in their learning capabilities (e.g., Bitterman, 1965; Gonzales, Behrend, \& Bitterman, 1967; Gossette, Gossette, \& Riddell, 1966; Gossette \& Krause, 1968; Johnson, Lyle, Edwards, $\&$ Penny, 1974). The principal procedure in these experiments has been to train the animals to select one of two alternative visual stimuli which are presented either simultaneously or successively. After attaining some criterion of learning, or completing an arbitrary number of trials, the animals are required to select the other alternative.

Differences in the learning abilities of three vertebrate classes (Mammalia, Aves, Osteichthyes) which emerged from these experiments have sparked debates over their possible neural causes. Goldfish will improve marginally, both with singly presented stimuli and in choice situations, and, according to some researchers, in ways unique to goldfish (Englehart, Woodard, \& Bitterman, 1973; Woodard \& Bitterman, 1972; Woodard, Schoel, \& Bitterman, 1971). Rats and birds improve well, relative to fish, and, according to Gonzales et al. (1967) (who found no improvement in fish), in ways different from fish. On the basis of these data, some researchers contend that the differences between fish, birds, and rats stem from qualitative neural differences between the three classes. Bitterman and his colleagues outline evidence which indicates that pigeons and rats improve their performance over successive reversals primarily by a process of proactive interference in which the animals develop either weaker dispositions to respond to the rewarded stimulus of the previous session (Bitterman, 1969) or weaker inhibition to respond to the unrewarded stimulus of the previous session (Woodard \& Bitterman, 1972). Fish perhaps improve more by a

This paper is Contribution 9 of the Laboratory of Animal Behavior at Colorado State University. Requests for reprints should be sent to Philip N. Lehner, Laboratory of Animal Behavior, Colorado State University, Fort Collins, Colorado 80523. change in the rate of error production within reversals (Englehart et al., 1973).

Although several avian species have been tested in reversal experiments (Gossette et al., 1966), Bitterman's theories concerning this class stem entirely from work with pigeons. To determine if these theories can be truly generalized to the class Aves as a whole, we decided to test a different avian species, the mallard duck. Several researchers have pointed out the necessity of maintaining procedural variations at a minimum before judgments on phyletic differences in reversal performance should be made (Gonzales, Berger, \& Bitterman, 1966; Miller, Hansen, \& Thomas, 1972). We therefore used a procedure similar to that employed by Gonzales et al. (1967).

\section{METHOD}

\section{Subjects}

The experimental subjects were two female and three male naive adult mallard ducks maintained at $85 \%$ of ad-lib weight during the experiment.

\section{Apparatus}

The apparatus was similar to that used by Gonzales et al. (1967). The operant conditioning chamber contained an intelligence panel with two $3-\mathrm{cm}$ circular stimulus patches located $10 \mathrm{~cm}$ apart and $20 \mathrm{~cm}$ above the floor. The arabic numerals 8 and 1 were rear projected onto these stimulus patches by two Lehigh Valley Electronics multistimulus rear-screen projectors. Directly below each stimulus patch was a pecking key. In this respect, the apparatus differed from that of Gonzales et al. (1967), in which red and green stimuli were projected directly on the pecking keys. A food tray was centered between and below the keys. Purina Layena pellets $(300 \mathrm{mg})$ served as reinforcers. House illumination was provided by a $71 / 2-W$ tungsten bulb located at the top of the test chamber. A continuous masking noise was provided by a BRS-Foringer audiogenerator. All events of the experiment were controlled and recorded automatically and were monitored by closed-circuit television.

\section{Procedure}

The test regime was identical to that of Gonzales et al. (1967). In a 2-day reversal situation, the animals were trained to discriminate between the numerals 8 and 1 presented simultaneously in a series of 40-trial daily sessions. The numeral 8 was the rewarded stimulus on odd pairs of days (e.g., 1, 2) and the 
numeral 1 was the rewarded stimulus on even pairs of days (e.g., 3, 4), continuing for a total of 40 reversals. Each trial began with the presentation of the two symbols, their positions varying randomly from trial to trial, and the illumination of the houselight. A response on the correct key turned off the two symbols for $6 \mathrm{sec}$ and dispensed a food pellet. An incorrect response turned off both the symbols and houselight for $6 \mathrm{sec}$, leaving the animal in darkness. Whenever four consecutive errors occurred, the correct symbol alone was illuminated until a correct response was given (correction procedure).

Measurements were taken of: (1) the number of errors per reversal, (2) the number of errors each day of a reversal, and (3) the intrasession error distribution.

\section{RESULTS AND DISCUSSION}

The overall performance of the mallards in terms of total errors per reversal is plotted in the upper curve of Figure 1 . The data were analyzed by a duck by day by reversal analysis of variance for factorial design. A definite course of improvement occurred $[\mathrm{F}(1,316)=79.23$, $\mathrm{p}<.05]$, but was not as rapid as that reported by Gonzales et al. (1967) for the pigeon.

The total errors which occurred during each of the 2 days of each reversal are plotted by the two lower curves of Figure 1. The two curves differed in the number of errors occurring in each $[F(39,316)=.61$, $\mathrm{p}<.05]$. This latter finding is in direct contrast to the results reported by Gonzales et al. (1967) for the pigeon in which more improvement occurred during the first day of each reversal.

The foregoing results for the pigeons suggested to previous researchers that the birds were improving their overall performance by developing weaker dispositions to respond to the correct stimulus of the previous day. As the pigeons began the sessions of Day 1 (the reversal day), they possessed progressively less retention of the pervious correct stimulus, and therefore improved markedly during this time. However, on Day 2 (the nonreversal day) this weakened retention caused a tendency to increase responses to the incorrect stimulus, and therefore overall improvement was less.

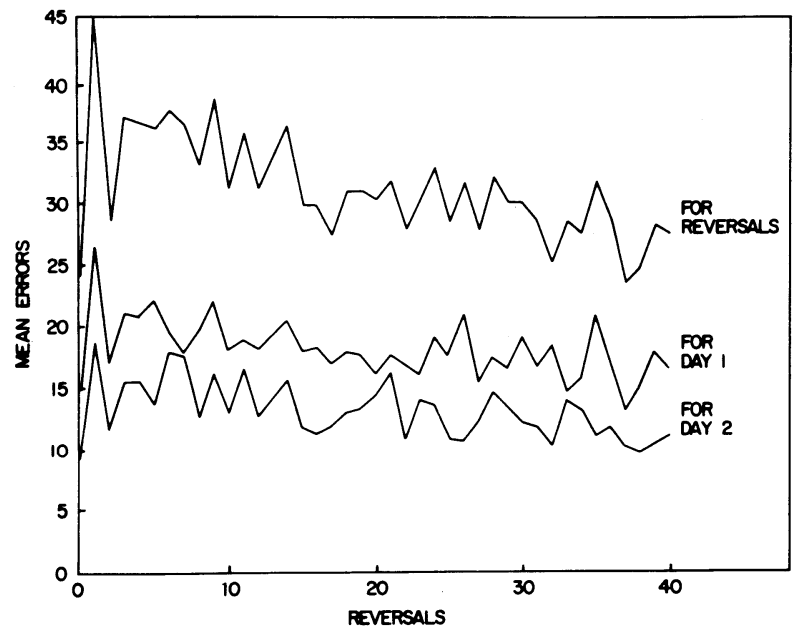

Figure 1. Mean error curves.

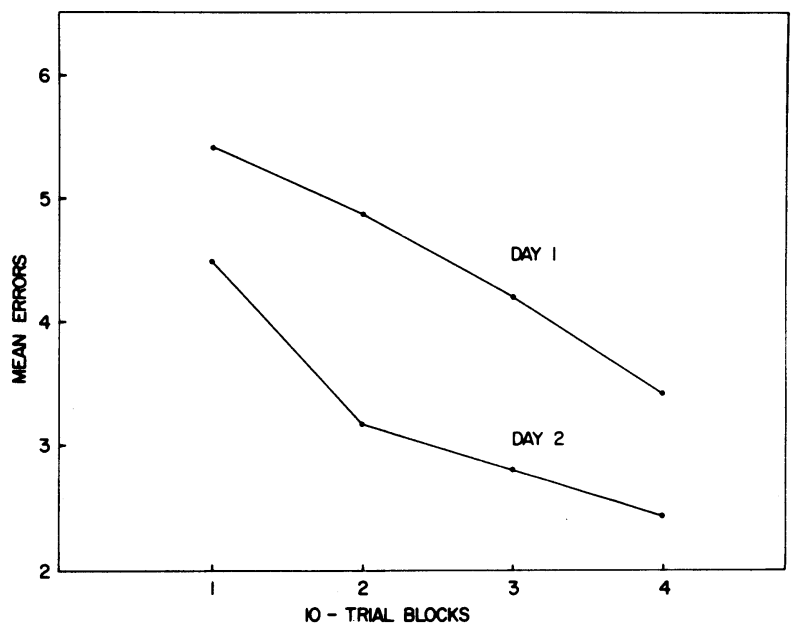

Figure 2. Mean intrasession error distribution.

Since the mallards did not seem to be improving in this manner, the data were analyzed in terms of initial errors for each reversal. The errors which occurred during the first trial of each reversal were tested across all 40 reversals, and no significant differences were found between them $[F(39,160)=.09, p<.05]$. The initial errors for Day 2 of each reversal were tested in the same way, and here again the differences were not significant $[\mathrm{F}(39,160)=.13, \mathrm{p}<.05]$.

The mean intrasession error distribution for each day of the 40 reversals is shown in Figure 2. The 40 trials which made up each daily session were divided into four 10-day trial blocks and the mean errors for each block were plotted for Day 1 and Day 2. A significant decrease in errors occurred for both days $[F(1,948)=5,497.24$, $p<.05]$. This result, coupled with the fact that there was not much change in initial preference across the 40 reversals, indicates on both days a relatively stable preference may have existed for the previous day's correct stimulus. Overall improvement must, then, have occurred as a decrease in the within-reversals error curve.

Since it has been shown that pigeons improve their performance in reversal experiments by developing either progressively weaker retention (Gonzales et al., 1967) or progressively stronger inhibitions (Woodard \& Bitterman, 1972), it has been previously suggested that birds improve by proactive interference. Fish have not given evidence of proactive interference. Therefore, it has also been previously suggested that fish and birds differ qualitatively in their neural mechanisms.

While this may be true in comparisons between pigeons and fish, it may not be the case with mallards. The ducks did not appear to improve by developing progressively weaker retention, but rather showed progressive improvement in repetitive errors. It is not possible to state this difference between mallards and pigeons with certainty. Since the mallards improved so poorly overall relative to pigeons, other factors involving 
the experimental design may have been determinant. The ease of discriminating the red and green stimuli of the Gonzales et al. (1967) experiment may not be equivalent to the ease of discriminating the symbols 8 and 1 of the present experiment. However, other researchers have also described improvement in various birds which could not be attributable to forgetting (Gossette, 1968; Mackintosh, 1969). Since their procedures differed markedly, these results cannot be compared directly with either the mallards or the pigeons, but it is apparent other mechanisms are involved in the reversal learning of some birds. While this does not indicate the lack of other qualitative neural differences between mallards and fish, it does point out the necessity of obtaining data from a greater variety of avian species, using a uniform procedure.

\section{REFERENCES}

Bitterman, M. E. Phyletic differences in learning. American Psychologist, 1965, 20, 396-410.

BitTerman, M. E. Habit reversal and probability learning: Rats, birds and fish. In R. M. Gilbert \& N. S. Sutherland (Eds.), Animal discrimination learning. New York: Academic Press, 1969. Pp. 163-174.

Englehart, F., Woodard, W. T., \& Bitterman, M. E. Discrimination reversal in the goldfish as a function of training conditions. Journal of Comparative and Physiological Psychology, 1973, 85, 144-150.

Gonzales, R. C., Berger, B. D., \& Bitterman, M. E. Improvement in habit-reversal as a function of amount of training per reversal and other variables. American Journal of Psychology, 1966, 79, 517-530.
Gonzales, R. C., Behrend, E. R., \& Bitterman, M. E. Reversal learning and forgetting in bird and fish. Science, 1967, 158, 519-521.

Gossette, R. L. Examination of retention decrement explanation of comparative successive discrimination reversal learning by birds and mammals. Perceptual and Motor Skills, 1968, 27, 1147-1152.

Gossette, R. L., Gossette, M. F., \& Riddell, W. Comparisons of successive discrimination reversal performances among closely and remotely related avian species. Animal Behavior, 1966, 14, 560-564.

Gossette, R. L., \& Krause, G. Successive discrimination reversal performance of mammalian species on a brightness task. Perceptual and Motor Skills, 1968, 27, 675-678.

Jonson, K. M., Lyle, J. G., Edwards, M. J., \& Penry, R. H. C. Spatial and nonspatial discrimination reversal (SDR) learning in the guinea pig. Animal Behavior, 1974, 22, 118-123.

Mackintosh, N. J. Habit reversal and probability learning: Rats, birds, and fish. In R. M. Gilbert \& N. S. Sutherland (Eds.), Animal discrimination learning. New York: Academic Press, 1969. Pp. 175-184.

Miller, J. T., Hansen, G., \& Thomas, D. R. Effects of stimulus similarity and response criterion on successive discrimination learning. Journal of Comparative and Physiological Psychology, 1972, 81, 434-440.

Woodard, W. T., Schoel, W. M., \& Bitterman, M. E. Reversal learning with singly presented stimuli in pigeons and goldfish. Journal of Comparative and Physiological Psychology, 1971, 74, 460-467.

Woodard, W. T., \& Bitterman, M. E. Further studies of reversal learning with singly presented stimuli in pigeons and goldfish. Psychonomic Science, 1972, 28, 170-172.

(Received for publication May 31, 1977.) 